\title{
A 'silent' skull metastatic follicular thyroid carcinoma mimicking as a benign scalp tumor in a pregnant woman
}

\author{
Tsung-Chun Huang1, Yu-Kai Cheng², Tsung-Wei Chen ${ }^{3}$, Yung-Chang Hsu', \\ En-Wei Liu ${ }^{1}$ and Hsin-Han Chen ${ }^{1}$
}

Departments of ${ }^{1}$ Plastic and Reconstructive Surgery, ${ }^{2}$ Neurosurgery, and ${ }^{3}$ Pathology, China Medical University Hospital, China Medical University, Taichung, Taiwan

Correspondence
should be addressed
to H-H Chen
Email
scapulachenhh@yahoo.com.tw

\section{Summary}

Thyroid cancer with cranial metastasis in a pregnant woman is very rare. In the literature, most cases are diagnosed early from neurogenic signs or symptomatic thyroid gland. Pregnancy also contributes to a hesitation toward early surgical and medical treatments. We reported a scalp tumor in a physically healthy 37-year-old pregnant female with a follicular thyroid carcinoma (FTC) with lung, bone and cranial metastasis in initial presentation. Silent neurogenic and physical examinations make an early diagnosis very challenging. Resection of scalp and intracranial tumor, a thyroidectomy, post-operative radioactive iodine therapy and tyrosine kinase inhibitors were employed as treatment. The scalp tumor was confirmed as a metastatic follicular thyroid carcinoma via positive immunoreactivity for thyroglobulin and thyroid transcription factor 1 in tumor cells. Blood examination revealed an elevated thyroglobulin level ( $>5335 \mathrm{ng} / \mathrm{mL})$. The patient was discharged without any neurological deficit. An asymptomatic scalp tumor in a pregnant woman with a normal thyroid disease history needs differential diagnosis from intracranial origin. Rapid progression and an elevated thyroglobulin level are the indicators that further image study is needed. Aggressive surgical excision of resectable thyroid gland and metastatic tumor are essential for a longer survival rate. There is nothing to indicate that a postpartum operation will worsen prognosis.

\section{Learning points:}

- Follicular thyroid cancer with cranial metastasis in initial presentation can be asymptomatic.

- Follicular thyroid cancer with cranial metastasis in a pregnant woman can be treated after delivery.

- Rapid enlargement of scalp tumor is indicated for further image study even in a patient without any neurological deficit.

\section{Background}

Thyroid cancer is the second most common cancer diagnosed during pregnancy. Most cases are diagnosed early from physical examination, with symptoms being a swollen neck and/or palpable lymph nodes. Thyroid cancer with central nervous system metastasis rarely occurs, and it accounts for only about $1 \%$ of patients.
However, the incidence rate of thyroid cancer with brain metastasis as an initial presentation has not been described well. Few case reports have been written, but either positive physical and neurological signs or a malignant thyroid disease can be identified during history-taking. To our knowledge, thyroid carcinoma with multiple 
metastases in initial presentation in a pregnant woman, whose thyroid medical history is benign, has never been reported.

We have reported on this rare case in this article and reviewed the surrounding literature. We also have discussed the clinical dilemmas present and whether a patient should be treated after delivery or not.

\section{Case presentation}

A 37-year-old pregnant woman came to our clinic due to a left scalp tumor that was palpable when she washed her hair. Her medical history includes that her gestational age (GA) at the time was 21 weeks and that she had a thyroid follicular adenoma before which was treated with rightside subtotal thyroidectomy in a separate local hospital five years earlier. Before she discovered this oval-shaped tumor on her left scalp, there were no symptomatic signs, such as headache, pain, neck mass or chronic cough. Clinically, the scalp tumor measured about $3.8 \times 1.0 \mathrm{~cm}$ with sharp margins and was movable. As there are no history of thyroid carcinoma and no neurological deficit, pre-operative brain MRI does not meet cost-effectiveness (MRI is not covered by our health insurance system). Brain CT is also contra-indicated in a pregnant woman. Therefore, the only image study is ultrasonography before operation. The sonography revealed a homogenous tumor in the subcutaneous layer with clear margins. We followed up with this case until the patient delivered a healthy full-term infant.

During the third trimester, the tumor gradually increased to $7.0 \times 7.0$ in size. The tumor was soft and elastic with normal hair distribution and skin color. There was no neurological sign except left lower back pain found after delivery of the baby. There is no change in mental status, no headache, no facial nerve palsy, no focal weakness, no hemiparesis, no nausea and vomiting and no sensation disturbance.

A scheduled excisional biopsy of the tumor under general anesthesia was arranged after her delivery. A smooth dissection was performed above the galea plane, but hypervascularity with some engorged vessels were encountered around the base of the tumor (Fig. 1A). After resection, the scalp tumor with left parietal bone invasion was highly suspected to be correlated with malignant distant metastasis (Fig. 1B, C and D). The final pathologic report confirmed the diagnosis of a follicular carcinoma of thyroid origin (Fig. 2).

Lack of a well-prepared image study was the drawback in our study. Both family and doctors expected the
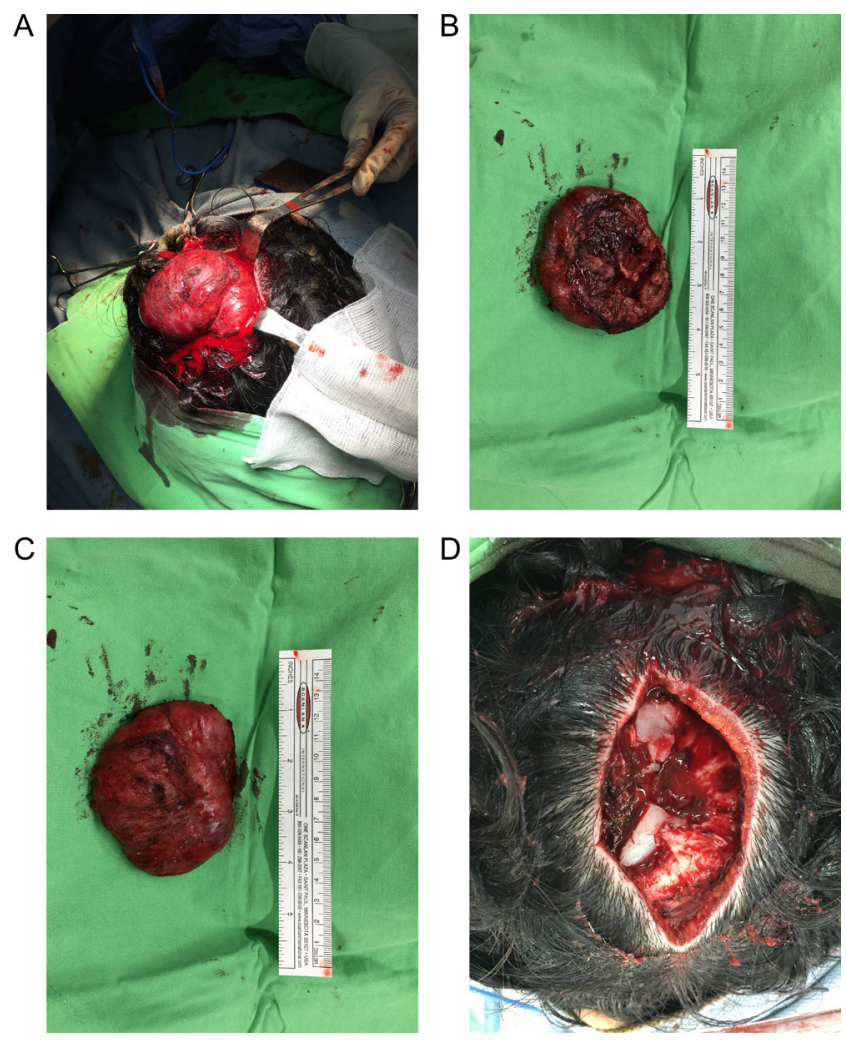

Figure 1

First stage of surgery for left scalp tumor. Smooth plane can be dissected above galea plane, but hypervascularity with some engorged vessels were encountered around the base of tumor (A). One $7.0 \times 7.0 \mathrm{~cm}$ tumor was resected. The surface of tumor (B) was smooth, but some necrotic tissue and bony fragment were identified from basal view (C). Skull bone bony defect highly suggests intracranial origin of this tumor (D).

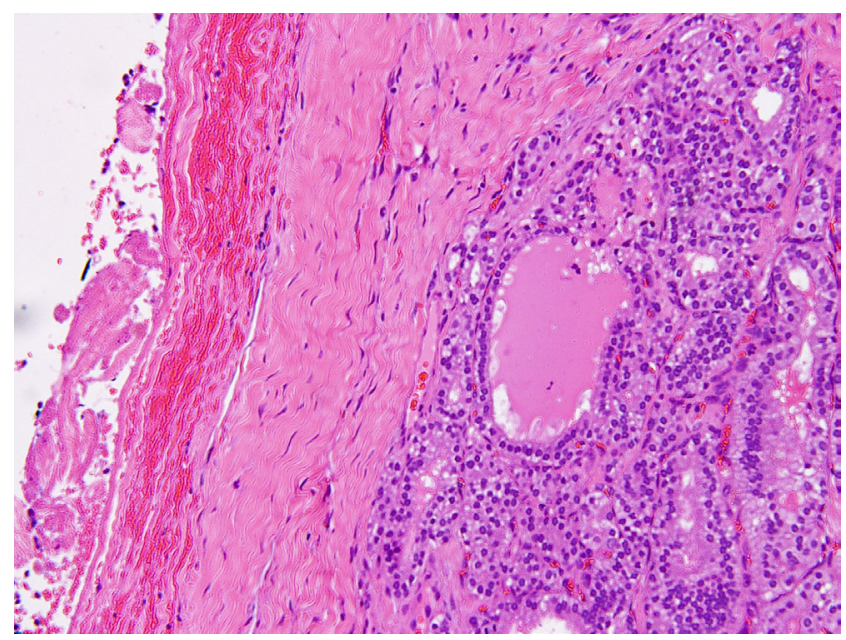

Figure 2

Frozen section of scalp tumor revealed that there are abundant cube-shaped cells accompanied with light eosinophilic colloid within follicles (H\&E stain, 200x). 
surgery to be a minor one, but it was not. During the first operation, blood loss was about $1500 \mathrm{~mL}$. A neurosurgeon was also not on stand-by initially. The pathologist could confirm the thyroid carcinoma metastasis via frozen section. Lack of an image study also made it difficult for the neurosurgeon to identify the true lesions because the operative field had massive oozing. We could not perform craniotomy first as we had to explain the severity of disease to the family and let the neurosurgeon come to a better decision after the results from permanent pathology and image reports were available.

General surgical consultations accompanying malignancy systemic evaluation were obtained. Contrastenhanced magnetic resonance imaging (MRI) revealed intracranial heterogenous, osteolytic and extra-axial mass located at left parietal bone area (Fig. 3), and it measured $7.3 \times 6.0 \times 7.0 \mathrm{~cm}$ in size. Thyroid sonography could not detect any mass lesions on either side. Free T 4 was $0.43 \mathrm{ng} / \mathrm{dL}$ (normal: $0.54-1.40$ ), free T3 was $64.49 \mathrm{ng} / \mathrm{dL}$ (normal: 70-170), thyroid-stimulating hormone was $0.47 \mathrm{U} / \mathrm{L}$ (normal: 0.34-5.60) and thyroglobulin was elevated to above $5335 \mathrm{ng} / \mathrm{mL}$ (normal: <55). A bone scan and a positron emission tomography scan revealed multiple bone metastases on the thoracic, lumbar spine, ilium and sacrum bones (Fig. 4A). In a chest CT scan, bilateral lung metastases were also identified.
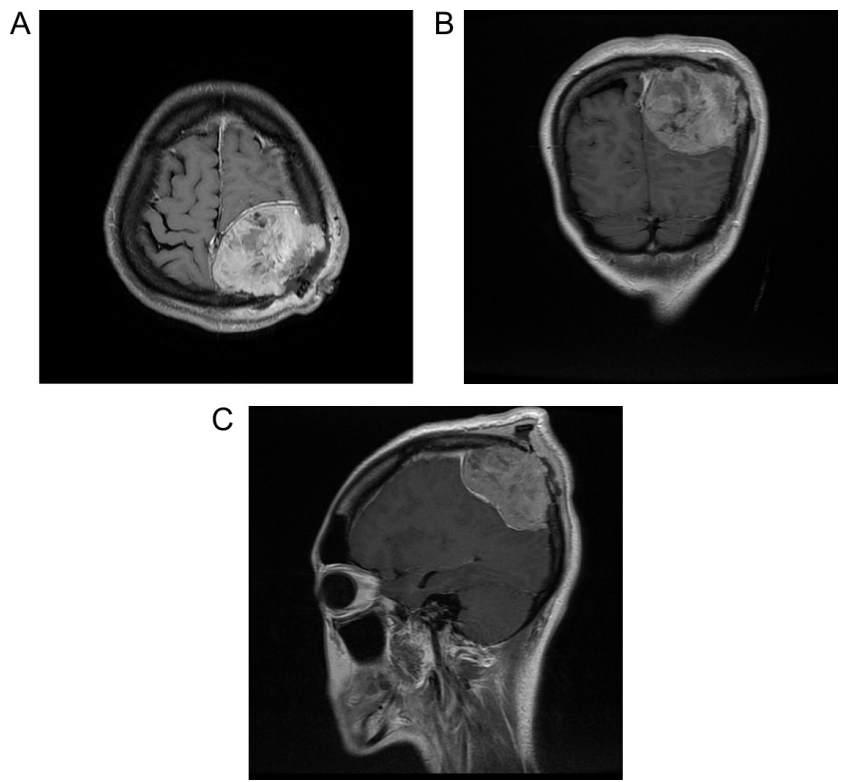

Figure 3

Contrast-enhanced magnetic resonance imaging (MRI) with contrast after first-stage scalp tumor removal. Horizontal (A), coronal (B) and sagittal (C) view revealed one heterogenous intracranial mass lesion with destroying left parietal and occipital bone skull bone.

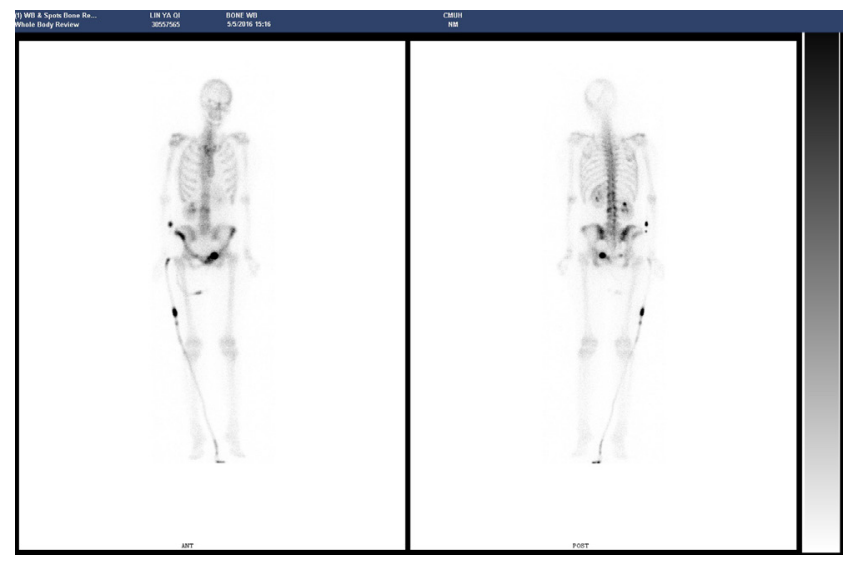

Figure 4

Bone scan revealed multiple bone metastases on thoracic, lumbar spine, ilium and sacrum bone.

After serial systemic evaluation, the patient underwent craniotomy surgery for the resection of the metastatic tumor. Intra-operatively, the metastatic tumor invaded the dura and arachnoid membrane without brain parenchyma involvement. Brain tissue was decompressed after ablation of the tumor, and brain tissue fully expanded without any tissue loss.

Immunohistochemistry showed positivity for thyroglobulin and thyroid transcription factor 1 (TTF-1), which established strong evidence for metastatic thyroid carcinoma origin (Fig. 5) (2). Two weeks after the craniectomy, however, a left thyroid lobectomy revealed that there was no residual follicular carcinoma inside.

The post-operative course went smoothly. Her muscle power was 5 in each side, and she was discharged on her own accord without nerve palsy or hemiplegia. She received levothyroxine therapy $150 \mu \mathrm{g}$ per day, and her free T4 level is within normal range. After post-operative recovery, a dose of 200 millicuries (mCi) of radioactive iodine (131I) (Fig. 6) and tyrosine kinase inhibitor were prescribed for her. Five months after the operation, she still survives and follows up regularly.

\section{Discussion}

To our knowledge, this is the first case where a silent multiple metastatic follicular thyroid carcinoma (FTC) presented in a pregnant woman without previous related medical history. The only clinical clue in this case was the patient's history of benign follicular adenoma (FA) informed at a local clinic five years. The patient received right subtotal thyroidectomy at that time. We asked the local hospital to review the initial diagnosis. However, the gross specimen is missing and the remaining 


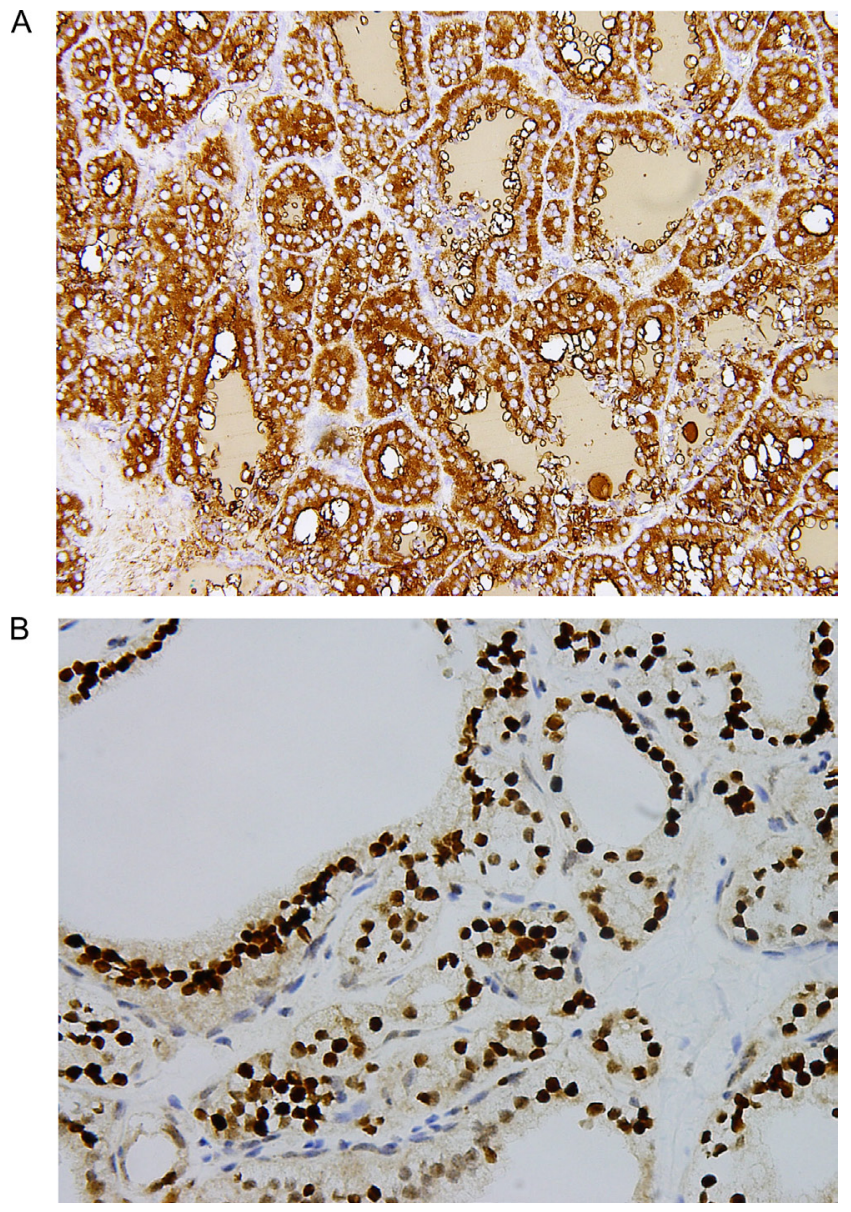

Figure 5

Positive findings for thyroglobulin (A) and thyroid transcription factor 1 (B) confirm the diagnosis of metastatic thyroid carcinoma

pathology slide still contributes to the diagnosis of thyroid adenoma. The erroneous diagnosis is probably due to the limitation of pathological diagnosis. From the point of view of histology, a minimally invasive follicular

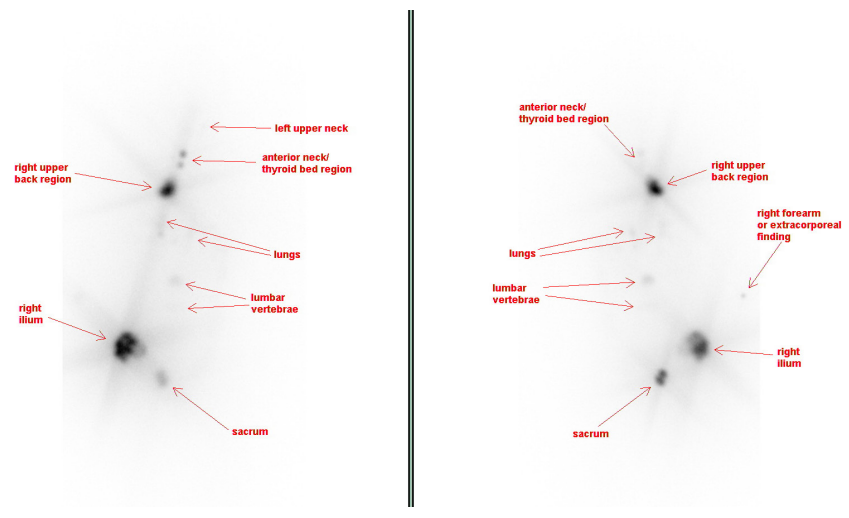

Figure 6

I131 whole body scan revealed increased radioactivity over bilateral lungs, upper back and pelvic bone area. thyroid carcinoma is difficult to be distinguished from a follicular adenoma of the thyroid. It is still unclear as to whether or not FTC arises from pre-existing FA or not. The diagnosis of minimally invasive FTC entirely depends on the demonstration of limited vascular invasion and/ or localized tumor invasion through its capsule. If the protrusion of thyroid capsule is not just cut and identified, the FTC will be considered as thyroid adenoma. Lee et al. have reported the average interval between the first diagnosis of thyroid cancer and intracranial metastasis was 50.5 months (range, 0-150 months) (3). Therefore, the patient's past medical information ought to be interpreted to mean that the correct pathological diagnosis at that time should be follicular thyroid carcinoma. In the literature review, there has been one case reported where a brain metastatic follicular thyroid carcinoma occurred without neurological signs in a 65-year-old female 12 years after initial presentation (2). Intracranial metastasis accounts for about $1 \%$ of thyroid malignancies (4). The fact that this case was a soft and elastic scalp tumor in a pregnant woman with silent neurological expression made the clinician not be alert to differentiate the possibility of the tumor's intracranial origin.

Distant metastasis (DM) is categorized into two groups: DM at initial presentation and DM after initial treatment (5). Older females are the predominant patient group in DM. The primary diagnosis of distant metastasis thyroid cancer is very rare, ranging from $1 \%$ to $7.2 \%(5,6,7)$. Due to its rarity, pre-operative diagnosis of DM is very challenging (8), especially in our case. Pregnancy at a GA of 21 weeks and limited medical history made the pre-operative diagnosis much more difficult. Thyroid cancer is the second most common newly diagnosed cancer during pregnancy (1). Some authors have correlated the progression of malignancy to the binding between human chorionic gonadotropin $(\beta$-HCG) and TSH receptors. This hypothetical mechanism probably explains the rapid progression of the tumor in our case (9). Indeed, the tumor grew two times in size from $3.8 \times 1.0 \mathrm{~cm}$ to $7.0 \times 7.0 \mathrm{~cm}$ during our patient's pregnancy. On the other hand, in a meta-analysis review, Negri et al. could not conclude if a strong association between reproductive factors and thyroid cancer exist. Only younger pregnant women diagnosed with papillary thyroid cancer may have higher risks (10). Some claim that estrogen-binding receptors could be identified on thyroid tumors (11), but this remains suspect as the results could not be replicated (12). Clarity on the mechanism between pregnancy and thyroid follicular carcinoma still requires more studies. 
This huge cranial metastatic tumor, however, did not have any neurological signs. To our knowledge, the only patient in the literature who had a similar clinical presentation without any neurological anomalies, had her tumor, like our patient, confined within the dura (2). The patient's lower back pain can be explained by pelvic bone metastasis, but this sign can be confused as pregnancy related. The most common DM is lung, followed by bone, and then brain $(3,5)$. Recently, the overall survival rate from the data, which shows great improvement over Haq et al., has been estimated at $83.8 \%$ and $72.1 \%$ for 5 and 10 years respectively (5). Cranial metastasis is regarded as a poor prognostic factor (3), whereas cranial metastasis without brain involvement has been seldom reported.

Optimal treatments for thyroid malignancy with metastasis in pregnancy are still not standardized due to its rarity. Maternal and neonatal morbidity and complications are the major concern (9). FTC is the second most common thyroid cancer after papillary thyroid carcinoma (PTC) during pregnancy, and the prevalence rate is about 14 per 100000 live births (1). Differing from the lymphatic route in PTC, FTC tends to metastasize via angioinvasion with an aggressive pattern. When compared with PTC, FTC displays a shorter period until intracranial metastasis (3). The reported average survival for patients with follicular carcinoma and brain metastasis is 8.3 months (7). However, pregnancy has been found to have no impact on the prognosis of women with thyroid cancer. There were no correlations found between maternal and neonatal complications in the study by Yasmeen's et al. The survival rate also shows no significant decrease in pregnant women compared to similarly aged non-pregnant women (9). Therefore, if a correct diagnosis of brain metastasis from thyroid origin is identified, as in our case, the pregnant woman's tumor progression still needs to be observed until her delivery. Our case in particular shows that further image studying can strongly indicate a rapidly progressive scalp tumor found during pregnancy, even a tumor without neurological symptoms.

Prognostic factors in previous studies include the male gender, old age, poorly differentiated histological variants, incomplete surgical resection of a primary tumor, multiple metastasis and poor response to radioactive iodine (RAI) treatment $(3,5,13)$. The independent factor improving survival rate is surgical resection to achieve complete local control $(3,5,7)$. Removal of the entire thyroid and possible metastasectomy are recommended because they will decrease the tumor loading and the amount of radioactive iodine (RAI) therapy needed $(3,7)$. RAI therapy is only beneficial for uptake scan positive in brain metastasis. Unfortunately, there are few cases resistant to radioactive iodine therapy in distant metastatic thyroid cancer. As the reported uptake rate of brain metastasis ranges only from $17 \%$ to $23 \%$ (7), adjuvant whole-brain radiation therapy, stereotactic radiosurgery, cytotoxic chemotherapy, biotherapy and tyrosine kinase inhibitors should be considered for patients resistant to RAI therapy. Doxorubicin is the most frequent chemotherapy agent, but only partial response rate of $22 \%$ can be achieved (14). Recently, tyrosine kinase inhibitors was applied to these kinds of refractory cases and showed its potential in treatments. Dowson et al. (15) report two cases with 4-year disease-free survival after sunitinib therapy. In the case series of M.D. Anderson Cancer Center (16), they report partial remission rate of $20 \%$, durable response rate of $66 \%$ and a clinical benefit rate of $80 \%$. Lung metastasis is a more favorable site, but bony metastasis responds poorly. During the post-operative period, thyroglobulin (TG) level is effective for detecting distant metastasis in thyroid cancer patients, especially in FTC with brain metastasis (3). The dramatically increased TG level in our case is another possible early detector for diagnosing before operation.

\section{Summary}

In the above we have reported a rare case with diffuse metastatic follicular carcinoma in a pregnant woman. This silent scalp tumor case forces us to confront possible malignancy even in patients with normal thyroid disease history. Rapid progression and an elevated thyroglobulin level are indicators of the need for further image study. Aggressive surgical excision of cranial metastasis should only be performed after delivery.

\section{Declaration of interest}

All authors certify that we have no affiliations with or involvement in any organization or entity with any financial interest (such as honoraria; educational grants; participation in speakers' bureaus; membership, employment, consultancies, stock ownership or other equity interest; and expert testimony or patent-licensing arrangements) or non-financial interest (such as personal or professional relationships, affiliations, knowledge or beliefs) in the subject matter or materials discussed in this manuscript.

\section{Funding}

None of the authors have any financial or personal relationships with persons or organizations that could inappropriately influence the study. 


\section{References}

1 Smith LH, Danielsen B, Allen ME \& Cress R 2003 Cancer associated with obstetric delivery: results of linkage with the California cancer registry. American Journal of Obstetrics and Gynecology 189 1128-1135. (doi:10.1067/S0002-9378(03)00537-4)

2 Ogawa Y, Sugawara T, Seki H \& Sakuma T 2006 Thyroid follicular carcinoma metastasized to the lung, skull, and brain 12 years after initial treatment for thyroid gland-case report. Neurologia MedicoChirurgica 46 302-305. (doi:10.2176/nmc.46.302)

3 Lee HS, Yoo H, Lee SH, Gwak HS \& Shin SH 2015 Clinical characteristics and follow-up of intracranial metastases from thyroid cancer. Acta Neurochirurgica 157 2185-2194. (doi:10.1007/s00701-015-2611-5)

4 Tagle P, Villanueva P, Torrealba G \& Huete I 2002 Intracranial metastasis or meningioma?: an uncommon clinical diagnostic dilemma. Surgical Neurology 58 241-245. (doi:10.1016/S00903019(02)00831-5)

5 Lee J \& Soh E-Y 2010 Differentiated thyroid carcinoma presenting with distant metastasis at initial diagnosis: clinical outcomes and prognostic factors. Annals of Surgery 251 114-119. (doi:10.1097/ SLA.0b013e3181b7faf6)

6 Haq M \& Harmer C 2005 Differentiated thyroid carcinoma with distant metastases at presentation: prognostic factors and outcome. Clinical Endocrinology 63 87-93. (doi:10.1111/j.13652265.2005.02304.x)

7 McWilliams RR, Giannini C, Hay ID, Atkinson JL, Stafford SL \& Buckner JC 2003 Management of brain metastases from thyroid carcinoma. Cancer 98 356-362. (doi:10.1002/cncr.11488)

8 Fujita T, Ogasawara Y \& Doihara H 2009 Solitary cranial metastasis of thyroid carcinoma 13 years after primary surgery: report of a case. Surgery Today 39 44-47. (doi:10.1007/s00595-008-3783-x)
9 Yasmeen S, Cress R, Romano PS, Xing G, Berger-Chen S, Danielsen B \& Smith LH 2005 Thyroid cancer in pregnancy. International Journal of Gynecology and Obstetrics 91 15-20. (doi:10.1016/j. ijgo.2005.06.022)

10 Negri E, Dal Maso L, Ron E, La Vecchia C, Mark SD, Preston-Martin S, McTiernan A, Kolonel L, Yoshimoto Y, Jin F, et al. 1999 A pooled analysis of case-control studies of thyroid cancer II. Menstrual and reproductive factors. Cancer Causes and Control 10 143-155. (doi:10.1023/A:1008880429862)

11 Imai Y, Yamakawa M, Matsuda M \& Takeshi K 1989 Endogenous sex hormone and estrogen binding activity in thyroid cancer. Histology and Histopathology 4 39-45.

12 Jaklic BR, Rushin J \& Ghosh BC 1995 Estrogen and progesterone receptors in thyroid lesions. Annals of Surgical Oncology $2429-434$. (doi:10.1007/BF02306376)

13 Lundgren CI, Hall P, Dickman PW \& Zedenius J 2006 Clinically significant prognostic factors for differentiated thyroid carcinoma. Cancer 106 524-531. (doi:10.1002/cncr.21653)

14 Baudin E \& Schlumberger M 2007 New therapeutic approaches for metastatic thyroid carcinoma. Lancet Oncology 8 148-156. (doi:10.1016/S1470-2045(07)70034-7)

15 Dawson S-J, Conus NM, Toner GC, Raleigh JM, Hicks RJ, McArthur G \& Rischin D 2008 Sustained clinical responses to tyrosine kinase inhibitor sunitinib in thyroid carcinoma. Anti-Cancer Drugs 19 547-552. (doi:10.1097/CAD.0b013e3282fc6cf7)

16 Cabanillas ME, Waguespack SG, Bronstein Y, Williams MD, Feng L, Hernandez M, Lopez A, Sherman SI \& Busaidy NL 2010 Treatment with tyrosine kinase inhibitors for patients with differentiated thyroid cancer: the MD Anderson experience. Journal of Clinical Endocrinology and Metabolism 95 2588-2595. (doi:10.1210/jc.2009-1923)

Received in final form 23 October 2016

Accepted 28 November 2016 